\title{
Compact operators whose adjoints factor through subspaces of $l_{p}$
}

\author{
by \\ Deba P. Sinha and Anil K. Karn (New Delhi)
}

\begin{abstract}
For $p \geq 1$, a subset $K$ of a Banach space $X$ is said to be relatively $p$ compact if $K \subset\left\{\sum_{n=1}^{\infty} \alpha_{n} x_{n}:\left\{\alpha_{n}\right\} \in \operatorname{Ball}\left(l_{p^{\prime}}\right)\right\}$, where $p^{\prime}=p /(p-1)$ and $\left\{x_{n}\right\} \in l_{p}^{\mathrm{s}}(X)$. An operator $T \in B(X, Y)$ is said to be $p$-compact if $T(\operatorname{Ball}(X))$ is relatively $p$-compact in $Y$. Similarly, weak $p$-compactness may be defined by considering $\left\{x_{n}\right\} \in l_{p}^{\mathrm{w}}(X)$. It is proved that $T$ is (weakly) $p$-compact if and only if $T^{*}$ factors through a subspace of $l_{p}$ in a particular manner. The normed operator ideals $\left(K_{p}, \kappa_{p}\right)$ of $p$-compact operators and $\left(W_{p}, \omega_{p}\right)$ of weakly $p$-compact operators, arising from these factorizations, are shown to be complete. It is also shown that the adjoints of $p$-compact operators are $p$-summing.

It is further proved that for $p \geq 1$ the identity operator on $X$ can be approximated uniformly on every $p$-compact set by finite rank operators, or in other words, $X$ has the $p$-approximation property, if and only if for every Banach space $Y$ the set of finite rank operators is dense in the ideal $K_{p}(Y, X)$ of $p$-compact operators in the factorization norm $\omega_{p}$. It is also proved that every Banach space has the 2-approximation property while for each $p>2$ there is a Banach space that fails the $p$-approximation property.
\end{abstract}

1. Introduction and notations. The approximation property of Banach spaces and its natural relationship with the theory of tensor products, studied by Grothendieck [5], draw heavily on his visualization of a relatively compact set as one sitting inside the convex hull of a norm null sequence of vectors. Surprisingly enough, the scope of such visualizations, viz. sets sitting inside the convex hulls of special type of null sequences, was never studied. On the other hand, definitions of special types of approximation properties that were studied later (e.g., $p$-approximation properties studied by Saphar [12] for $1 \leq p<\infty$ and by Reunov [9] for $0<p<\infty$ ) were always conceived via the tensor product route.

In this paper, hopefully in the spirit of A. Grothendieck, we define a $p$-compact set for $p \geq 1$ as one sitting inside the $p$-convex hull (to be defined later) of a $p$-summable sequence of vectors, and a $p$-compact operator as one that maps bounded sets to relatively $p$-compact sets. We study the

2000 Mathematics Subject Classification: Primary 46B20, 46B28, 46B50, 47B07.

Key words and phrases: compact operator, factorization, approximation property. 
factorization of $p$-compact operators. This factorization yields a complete norm $\kappa_{p}$ on the operator ideal $K_{p}$ of $p$-compact operators. We study the position of $\left(K_{p}, \kappa_{p}\right)$ in operator ideals and observe that the adjoints of $p$ compact operators are $p$-summing. The ideas of $p$-compact sets, $p$-compact operators and their factorization can be studied in a more general context, namely that of weak $p$-compactness. We show that the operator ideal $W_{p}$ of weakly $p$-compact operators is complete in the factorization norm $\omega_{p}$. It is interesting to note that $p$-summing operators map weakly $p$-compact sets to $p$-compact sets. Thus $p$-summing operators may be thought of as " $p$-completely continuous" maps.

We consider the approximation of the identity operator on $p$-compact sets by finite rank operators, that is, the $p$-approximation property (for short, $p$-a.p.). The classical approximation property is equivalent to the density of the finite rank operators $F(Y, X)$ in the ideal $K(Y, X)$ of compact operators for every Banach space $Y$ in the operator norm. Surprisingly, we prove that the $p$-a.p. on $X$ is equivalent to the $\omega_{p}$-density of $F(Y, X)$ in $K_{p}(Y, X)$. We show that every Banach space has the 2-a.p. while for each $p>2$ there is a Banach space that fails the $p$-a.p.

At the end of the paper we study the $p$-approximation properties due to Saphar [12] and Reinov [9] through the notion of $(q, p)$-approximation property, $1 \leq p \leq q \leq \infty$, which is weaker than the $p$-a.p.

We denote Banach spaces by $X$ and $Y$, the closed unit ball of $X$ by $\operatorname{Ball}(X)$, the dual of $X$ by $X^{*}$ and the space of all bounded linear operators from $X$ to $Y$ by $B(X, Y)$. In $B(X, Y)$ the subspaces of all finite rank and all compact operators are denoted by $F(X, Y)$ and $K(X, Y)$, respectively. Further the projective tensor product $X \widetilde{\otimes}_{\pi} Y$ of $X$ and $Y$ consists of all elements $\varphi$ having a representation of the form $\varphi=\sum_{n=1}^{\infty} x_{n} \otimes y_{n}$ with $\left\{x_{n}\right\} \subset X$ and $\left\{y_{n}\right\} \subset Y$ such that $\sum_{n=1}^{\infty}\left\|x_{n}\right\| \cdot\left\|y_{n}\right\|<\infty$. The projective norm of $\varphi$ is the infimum of $\sum_{n=1}^{\infty}\left\|x_{n}\right\| \cdot\left\|y_{n}\right\|$ over all such representations. For any operator $T \in B(X, Y), N(T)$ and $R(T)$ denote the null space and range of $T$ respectively. Finally, for a bounded sequence $\left\{x_{n}\right\}$ in $X$ the convex hull of $\left\{x_{n}\right\}$ is denoted by $\operatorname{conv}\left\{x_{n}\right\}$ and is given by

$$
\operatorname{conv}\left\{x_{n}\right\}=\left\{\sum_{n=1}^{\infty} \alpha_{n} x_{n}: 0 \leq \alpha_{n} \leq 1, \sum_{n=1}^{\infty} \alpha_{n}=1\right\} .
$$

2. Compact operators - expanding the scope. Let us recall the following characterization of relatively compact sets in Banach spaces: A subset $K$ of a Banach space $X$ is relatively compact if and only if there is a null sequence $\left\{x_{n}\right\}$ in $X$ such that $K \subset \operatorname{conv}\left\{x_{n}\right\}$. Moreover, given $\varepsilon>0$ we can choose $\left\{x_{n}\right\}$ such that $\sup \{\|x\|: x \in K\}+\varepsilon>\sup \left\{\left\|x_{n}\right\|: n \in \mathbb{N}\right\}$. Expanding the scope, in order to study particular types of compact sets and 
allied notions, it may be assumed that $\left\{x_{n}\right\}$ is $p$-summable for some $p>0$. This is the main purpose of this paper. To this end, we find it desirable to modify the notion of convex hull. We start with the following concepts.

$A$ Tool Box. Let $X$ be a Banach space, $p \geq 1$ and let $l_{p}^{\mathrm{w}}(X)$ denote the set of all weakly $p$-summable sequences in $X$. Then $l_{p}^{\mathrm{w}}(X)$ is a Banach space with the norm

$$
\left\|\left\{x_{n}\right\}\right\|_{p}^{\mathrm{w}}=\sup \left\{\left\|\left\{f\left(x_{n}\right)\right\}\right\|_{p}: f \in \operatorname{Ball}\left(X^{*}\right)\right\} .
$$

If $p>1$, then $l_{p}^{\mathrm{w}}(X)$ is isometrically isomorphic to $B\left(l_{p^{\prime}}, X\right)$ where $p^{\prime}=$ $p(p-1)^{-1}$. For $p=1, l_{1}^{\mathrm{w}}(X)$ is isometrically isomorphic to $B\left(c_{0}, X\right)$. Let these isometries be denoted by $E: l_{p}^{\mathrm{w}}(X) \rightarrow B\left(l_{p^{\prime}}, X\right)$ (resp. $E: l_{1}^{\mathrm{w}}(X) \rightarrow$ $\left.B\left(c_{0}, X\right)\right), \mathbf{x}\left(=\left\{x_{n}\right\}\right) \mapsto E_{\mathbf{x}}$, where $E_{\mathbf{x}}\left(\left\{\alpha_{n}\right\}\right)=\sum_{n=1}^{\infty} \alpha_{n} x_{n}$. Write $p$-conv $\mathbf{x}:=E_{\mathbf{x}}\left(\operatorname{Ball}\left(l_{p^{\prime}}\right)\right)$ for $1 \leq p<\infty$ and $\infty$-conv $\left\{x_{n}\right\}:=\operatorname{abs-conv}\left\{x_{n}\right\}$ $:=E_{\mathbf{x}}\left(\operatorname{Ball}\left(l_{1}\right)\right)$, the absolute convex hull of $\left\{x_{n}\right\}$. (Here $l_{p^{\prime}}=\left(l_{p}\right)^{*}$.)

Next, let $l_{p}^{\mathrm{s}}(X)$ be the subspace of $l_{p}^{\mathrm{w}}(X)$ of all $p$-summable sequences in $X(1 \leq p \leq \infty)$ and $c_{0}^{\mathrm{s}}(X)$ the space of all norm null sequences in $X$. Then $l_{p}^{\mathrm{s}}(X)$ is a Banach space with the norm

$$
\left\|\left\{x_{n}\right\}\right\|_{p}^{\mathrm{s}}=\left(\sum_{n=1}^{\infty}\left\|x_{n}\right\|^{p}\right)^{1 / p}, \quad 1 \leq p<\infty .
$$

Also $l_{\infty}^{\mathrm{s}}(X)$ is a Banach space in the norm

$$
\left\|\left\{x_{n}\right\}\right\|_{\infty}^{\mathrm{s}}\left(=\left\|\left\{x_{n}\right\}\right\|_{\infty}^{\mathrm{w}}\right)=\sup _{n \in \mathbb{N}}\left\|x_{n}\right\| .
$$

Moreover, $c_{0}^{\mathrm{s}}(X)$ is a closed subspace of $l_{\infty}^{\mathrm{s}}(X)$ in the above norm. We write $E\left(l_{p}^{\mathrm{s}}(X)\right)=E_{p}(X)(1 \leq p \leq \infty)$ and $E\left(c_{0}^{\mathrm{s}}(X)\right)=E_{0}(X)$. Note that $l_{1}^{\mathrm{s}}(X) \subset$ $l_{p}^{\mathrm{s}}(X) \subset l_{q}^{\mathrm{s}}(X) \subset c_{0}^{\mathrm{s}}(X)$ if $1 \leq p \leq q<\infty$.

Redefining compact sets. Let $K$ be a relatively compact set in a Banach space $X$ and let $\varepsilon>0$. We can find $\mathbf{x}=\left\{x_{n}\right\} \in c_{0}^{\mathrm{s}}(X)$ with $\left\|x_{n}\right\|_{\infty}<$ $\sup \{\|x\|: x \in K\}+\varepsilon$ such that $K \subset \operatorname{abs-conv}\left\{x_{n}\right\}=E_{\mathbf{x}}\left(\operatorname{Ball}\left(l_{1}\right)\right)$. Put $\bar{x}_{n}=\left\|x_{n}\right\|^{-1} x_{n}$ (if $x_{n} \neq 0$, otherwise $\bar{x}_{n}=0$ ) and let $\lambda_{n}=\left\|x_{n}\right\|$. Then $\overline{\mathbf{x}}=\left\{\bar{x}_{n}\right\} \in \operatorname{Ball}\left(l_{\infty}(X)\right)\left(\right.$ or $\left\{\bar{x}_{n}\right\}$ is a sequence in $\left.\operatorname{Ball}(X)\right)$ and $\boldsymbol{\lambda}=\left\langle\lambda_{n}\right\rangle \in$ $c_{0}$ with $K \subset E_{\overline{\mathbf{x}}} M_{\boldsymbol{\lambda}}\left(\operatorname{Ball}\left(l_{1}\right)\right)$ where $M_{\boldsymbol{\lambda}}: l_{1} \rightarrow l_{1}$ is given by $M_{\boldsymbol{\lambda}}(\boldsymbol{\alpha})=$ $\left\langle\lambda_{n} \alpha_{n}\right\rangle, \boldsymbol{\alpha}=\left\langle\alpha_{n}\right\rangle \in l_{1}$. Thus $K \subset\left\{\sum_{n=1}^{\infty} \alpha_{n} \bar{x}_{n}:\left\langle\alpha_{n}\right\rangle \in M_{\boldsymbol{\lambda}}\left(\operatorname{Ball}\left(l_{1}\right)\right)\right\}$ with $\left\{\bar{x}_{n}\right\} \in \operatorname{Ball}\left(l_{\infty}(X)\right)$. Next, let $\boldsymbol{\lambda} \in l_{p}\left(1 \leq p<\infty\right.$, so that $\left.\boldsymbol{\lambda} \in c_{0}\right)$. Then $M_{\lambda}\left(l_{p^{\prime}}\right) \subset l_{1}$ if $p \geq 1$ where $l_{p^{\prime}}=\left(l_{p}\right)^{*}$.

Definition 2.1. Let $X$ be a Banach space and $K \subset X$. Then $K$ is said to be relatively $p$-compact $(1 \leq p \leq \infty)$ if given $\varepsilon>0$ there is a sequence $\left\{\bar{x}_{n}\right\}$ in $\operatorname{Ball}(X)$ and $\boldsymbol{\lambda} \in l_{p}\left(\lambda \in c_{0}\right.$ if $\left.p=\infty\right)$ with $\|\boldsymbol{\lambda}\|_{p}<\sup \{\|x\|$ : $x \in K\}+\varepsilon$ such that $K \subset\left\{\sum_{n=1}^{\infty} \alpha_{n} \bar{x}_{n}:\left\langle\alpha_{n}\right\rangle \in M_{\boldsymbol{\lambda}}\left(\operatorname{Ball}\left(l_{p^{\prime}}\right)\right)\right\}$, where $l_{p^{\prime}}=\left(l_{p}\right)^{*}(1 \leq p<\infty)$ and $l_{p^{\prime}}=l_{1}$ if $p=\infty$. 
Alternatively, $K$ is said to be relatively $p$-compact, $1 \leq p \leq \infty$, if there is $\mathbf{x}=\left\{x_{n}\right\} \in l_{p}^{\mathrm{s}}(X)(1 \leq p<\infty)\left(\mathbf{x}=\left\{x_{n}\right\} \in c_{0}^{\mathrm{s}}(X)\right.$ if $\left.p=\infty\right)$ such that $K \subset E_{\mathbf{x}}\left(\operatorname{Ball}\left(l_{p^{\prime}}\right)\right)$, where $l_{p^{\prime}}$ is suitably defined. Note that the $\infty$-compact sets are precisely the compact sets and that $p$-compact sets are $q$-compact if $1 \leq p \leq q \leq \infty$. Furthermore, it is easy to see that in an arbitrary Banach space, for $1 \leq p<q \leq \infty$, a $q$-compact set may not be $p$-compact.

Definition 2.2. For Banach spaces $X, Y$ and $1 \leq p \leq \infty$, an operator $T \in B(X, Y)$ is said to be $p$-compact if $T$ maps bounded subsets of $X$ to relatively $p$-compact subsets of $Y$. In other words there is a $\mathbf{y} \in l_{p}^{\mathrm{s}}(Y)$ $\left(\mathbf{y} \in c_{0}^{\mathrm{s}}(Y)\right.$ if $\left.p=\infty\right)$ such that $T(\operatorname{Ball}(X)) \subset E_{\mathbf{y}}\left(\operatorname{Ball}\left(l_{p^{\prime}}\right)\right)$.

Parallel to these concepts we define weakly (relatively) $p$-compact sets and weakly $p$-compact operators as follows:

Definition 2.3. Let $X$ be a Banach space and $K \subset X$. Then $K$ is said to be relatively weakly $p$-compact $(1 \leq p<\infty)$ if there is $\mathbf{x} \in l_{p}^{\mathrm{w}}(X)$ such that $K \subset E_{\mathbf{x}}\left(l_{p^{\prime}}\right)\left(l_{p^{\prime}}=\left(l_{p}\right)^{*}\right)$, and $K$ is said to be relatively weakly $\infty$-compact if there is $\mathbf{x} \in c_{0}^{\mathrm{w}}(X)$ such that $K \subset E_{\mathbf{x}}\left(\operatorname{Ball}\left(l_{1}\right)\right)$.

Definition 2.4. For Banach spaces $X, Y$ and $p \geq 1$, an operator $T \in$ $B(X, Y)$ is said to be weakly p-compact if there is a $\mathbf{y} \in l_{p}^{\mathrm{w}}(Y)$ such that $T(\operatorname{Ball}(X)) \subset E_{\mathbf{y}}\left(\operatorname{Ball}\left(l_{p^{\prime}}\right)\right)$.

Let $K_{p}(X, Y)$ denote the set of $p$-compact operators and $W_{p}(X, Y)$ the set of weakly $p$-compact operators in $B(X, Y), 1 \leq p \leq \infty$. Note that for $1 \leq$ $p \leq q \leq \infty, K_{p}(X, Y) \subset K_{q}(X, Y), W_{p}(X, Y) \subset W_{q}(X, Y)$ and $K_{p}(X, Y) \subset$ $W_{p}(X, Y)$. Moreover, $E_{p}(X) \subset K_{p}\left(l_{p^{\prime}}, X\right)$ where $l_{p^{\prime}}$ is the predual of $l_{p}$, $1 \leq p<\infty$ and $E_{0}(X) \subset K\left(l_{1}, X\right)$ for all Banach spaces $X$. Again, note that $W_{p}\left(l_{p^{\prime}}, X\right)=B\left(l_{p^{\prime}}, X\right)$ where $l_{p^{\prime}}$ is the predual of $l_{p}, 1 \leq p<\infty$ $\left(l_{p^{\prime}}=c_{0}\right.$ if $\left.p=1\right)$.

3. Factorization of $p$-compact operators. Throughout this section we fix $p, 1 \leq p<\infty$. Let $X$ be a Banach space and $\mathbf{x} \in l_{p}^{\mathrm{w}}(X)$. Then $E_{\mathbf{x}} \in$ $W_{p}\left(l_{p^{\prime}}, X\right)=B\left(l_{p^{\prime}}, X\right)$, where $l_{p^{\prime}}$ is the predual of $l_{p}$ (i.e., $l_{p^{\prime}}=c_{0}$ if $p=1$ ). Let $N(\mathbf{x})$ denote the null space of $E_{\mathbf{x}}$. If $q_{\mathbf{x}}$ denotes the quotient map from $l_{p^{\prime}}$ to $l_{p^{\prime}} / N(\mathbf{x})$ and if $\widetilde{E}_{\mathbf{x}}$ is the corresponding operator in $B\left(l_{p^{\prime}} / N(\mathbf{x}), X\right)$, then we have $E_{\mathbf{x}}=\widetilde{E}_{\mathbf{x}} q_{\mathbf{x}}$. This idea can be generalized to weakly $p$-compact operators.

Let $X$ and $Y$ be Banach spaces and let $T \in W_{p}(X, Y)$. Find $\mathbf{y} \in l_{p}^{\mathrm{w}}(Y)$ such that $T(\operatorname{Ball}(X)) \subset E_{\mathbf{y}}\left(\operatorname{Ball}\left(l_{p^{\prime}}\right)\right)$. Let $x \in \operatorname{Ball}(X)$. Then there is an $\boldsymbol{\alpha} \in \operatorname{Ball}\left(l_{p^{\prime}}\right)$ such that $T x=E_{\mathbf{y}}(\boldsymbol{\alpha})$. If there is another $\boldsymbol{\beta} \in \operatorname{Ball}\left(l_{p^{\prime}}\right)$ with $E_{\mathbf{y}}(\boldsymbol{\beta})=T x$, then $\boldsymbol{\alpha}-\boldsymbol{\beta} \in N(\mathbf{y})$. Thus each $x \in X$ determines an $\boldsymbol{\alpha} \in l_{p^{\prime}}$ contractively (i.e., $\|\boldsymbol{\alpha}\|_{p^{\prime}} \leq\|x\|$ ), which is also unique up to $N(\mathbf{y})$. In this way, we obtain an operator $T_{\mathbf{y}}: X \rightarrow l_{p^{\prime}} / N(\mathbf{y})$ uniquely determined by 
$T$ and $\mathbf{y}$ with the property that for each $x \in X$ there is an $\boldsymbol{\alpha} \in l_{p^{\prime}}$ with $\|\boldsymbol{\alpha}\|_{p^{\prime}} \leq\|x\|$ such that $T_{\mathbf{y}}(x)=\boldsymbol{\alpha}+N(\mathbf{y})$. In particular, $T_{\mathbf{y}}$ is a contraction. Note that $N\left(T_{\mathbf{y}}\right)=N(T)$. We shall call $T_{\mathbf{y}}$ the essential quotient of $T$ with respect to $\mathbf{y}$. It is now immediate that $T=\widetilde{E}_{\mathbf{y}} T_{\mathbf{y}}$. We shall say, in such a case, that $T$ is quotiented in $l_{p^{\prime}}$ by $\mathbf{y} \in l_{p}^{\mathrm{w}}(Y)$. Thus $T \in B(X, Y)$ is quotiented in $l_{p^{\prime}}$ by some $\mathbf{y} \in l_{p}^{\mathrm{w}}(Y)$ if and only if $T \in W_{p}(X, Y)$.

Next, note that $N(\mathbf{y})^{\perp}$ can be identified with $\overline{R\left(E_{\mathbf{y}}^{*}\right)}$. It follows that $T^{*}$ factors through a subspace $R(\mathbf{y})=\overline{R\left(E_{\mathbf{y}}^{*}\right)}$ of $l_{p}$. More precisely, we have $T^{*}=S_{\mathbf{y}} E_{\mathbf{y}}^{*}$. Here $E_{\mathbf{y}}^{*}: Y^{*} \rightarrow R(\mathbf{y}) \subset l_{p}$ is given by $E_{\mathbf{y}}^{*}(f)=\left\langle f\left(y_{n}\right)\right\rangle, f \in Y^{*}$. Also, $S_{\mathbf{y}}: R(\mathbf{y}) \rightarrow X^{*}$ is given by $S_{\mathbf{y}}\left(\left\langle f\left(x_{n}\right)\right\rangle\right)=T^{*} f, f \in Y^{*}$. It follows that $S_{\mathbf{y}}$ can be identified with $T_{\mathbf{y}}^{*}$ and $E_{\mathbf{y}}^{*}$ with $\widetilde{E}_{\mathbf{y}}^{*}$ in the identification of $N(\mathbf{y})^{\perp}$ with $R(\mathbf{y})$. Hence, the above leads us to the following characterization of weakly $p$-compact operators.

Theorem 3.1. Let $X$ and $Y$ be Banach spaces, $1 \leq p<\infty$ and $T \in$ $B(X, Y)$. Then the following statements are equivalent:

(a) $T$ is weakly p-compact.

(b) $T$ is quotiented in $l_{p^{\prime}}$ by some $\mathbf{y} \in l_{p}^{\mathrm{w}}(Y)$.

(c) There are $\mathbf{y} \in l_{p}^{\mathrm{w}}(Y)$ and $S_{\mathbf{y}} \in B\left(R(\mathbf{y}), X^{*}\right)$ such that $T^{*}=S_{\mathbf{y}} E_{\mathbf{y}}^{*}$.

With little modifications in the proof of the above theorem, similar characterizations for $p$-compact operators can be obtained:

Theorem 3.2. Let $X$ and $Y$ be Banach spaces, $1 \leq p \leq \infty$ and $T \in$ $B(X, Y)$. Then the following statements are equivalent:

(a) $T$ is p-compact.

(b) $T$ is quotiented in $l_{p^{\prime}}$ by some $\mathbf{y} \in l_{p}^{\mathrm{s}}(Y)$.

(c) There are $\mathbf{y} \in l_{p}^{\mathrm{s}}(Y)$ and $S_{\mathbf{y}} \in B\left(R(\mathbf{y}), X^{*}\right)$ such that $T^{*}=S_{\mathbf{y}} E_{\mathbf{y}}^{*}$.

(d) There is a sequence $\overline{\mathbf{y}} \in \operatorname{Ball}\left(l_{\infty}(Y)\right), \boldsymbol{\lambda} \in l_{p}$ and $S_{\mathbf{y}} \in B\left(R(\mathbf{y}), X^{*}\right)$ such that $T^{*}=S_{\mathbf{y}} M_{\lambda} E_{\overline{\mathbf{y}}}^{*}$. Here $\mathbf{y}=\left\{\lambda_{n} \bar{y}_{n}\right\} \in l_{p}^{\mathrm{s}}(Y)$.

4. The operator ideal of $p$-compact operators. Let $X, Y$ and $Z$ be Banach spaces and $p \geq 1$. If $\mathbf{x} \in l_{p}^{\mathrm{w}}(X)$, then for any $S \in B(X, Y)$ we have $S E_{\mathbf{x}}=E_{S \mathbf{x}}$, where $S \mathbf{x}=\left\{S x_{n}\right\}$ if $\mathbf{x}=\left\{x_{n}\right\}$. Thus $S_{\mathbf{x}} \in l_{p}^{\mathbf{w}}(Y)$. Again if $T \in B(Z, Y)$, then $E_{\mathbf{x}} T(\operatorname{Ball}(Z)) \subset\|T\| E_{\mathbf{x}}(\operatorname{Ball}(X))$. Now, it follows that $W_{p}(X, Y)$ is an operator ideal. In the same manner it may be shown that $K_{p}(X, Y)$ is also an operator ideal.

Next, using the factorization of weakly $p$-compact and $p$-compact operators we obtain norms on $W_{p}(X, Y)$ and $K_{p}(X, Y)$ as follows: For $T \in$ $W_{p}(X, Y)$ define

$$
\begin{aligned}
\omega_{p}(T) & =\inf \left\{\left\|S_{\mathbf{y}}\right\| \cdot\left\|E_{\mathbf{y}}\right\|: T^{*}=S_{\mathbf{y}} E_{\mathbf{y}}^{*} \text { in Theorem 3.1(c) }\right\} \\
& =\inf \left\{\left\|S_{\mathbf{y}}\right\| \cdot\|\mathbf{y}\|_{p}^{\mathbf{w}}: T^{*}=S_{\mathbf{y}} E_{\mathbf{y}}^{*} \text { in Theorem 3.1(c) }\right\} .
\end{aligned}
$$


Similarly, for $T \in K_{p}(X, Y)$ we define

$$
\begin{aligned}
\kappa_{p}(T) & =\inf \left\{\left\|S_{\mathbf{y}}\right\| \cdot\left\|M_{\boldsymbol{\lambda}}\right\| \cdot\left\|E_{\overline{\mathbf{y}}}\right\|: T^{*}=S_{\mathbf{y}} M_{\lambda} E_{\overline{\mathbf{y}}}^{*} \text { in Theorem 3.2(d) }\right\} \\
& =\inf \left\{\left\|S_{\mathbf{y}}\right\| \cdot\|\boldsymbol{\lambda}\| \cdot\|\overline{\mathbf{y}}\|_{\infty}: T^{*}=S_{\mathbf{y}} M_{\lambda} E_{\overline{\mathbf{y}}}^{*} \text { in Theorem 3.2(d) }\right\} \\
& =\inf \left\{\left\|S_{\mathbf{y}}\right\| \cdot\|\mathbf{y}\|_{p}^{\mathrm{S}}: T^{*}=S_{\mathbf{y}} E_{\mathbf{y}}^{*} \text { in Theorem 3.2(c) }\right\} .
\end{aligned}
$$

It is immediate that for $T \in K_{p}(X, Y) \subset W_{p}(X, Y)$ we have $\|T\| \leq$ $\omega_{p}(T) \leq \kappa_{p}(T)$. In general, the two norms are different and even nonequivalent.

Theorem 4.1. $\left(W_{p}(X, Y), \omega_{p}\right)$ is a Banach operator ideal.

Proof. It is easy to see that for $T \in W_{p}(X, Y)$, we have $\omega_{p}(S T U) \leq$ $\|S\| \omega_{p}(T)\|U\|$ for any operators $S \in B(Y, Z), U \in B(W, X)$ and for every choice of Banach spaces $Z$ and $W$.

Let $\left\{T_{n}\right\}$ be a sequence in $W_{p}(X, Y)$ with $\sum_{n=1}^{\infty} \omega_{p}\left(T_{n}\right)<\infty$. Then $\sum_{n=1}^{\infty}\left\|T_{n}\right\|<\infty$, and consequently we find a $T \in B(X, Y)$ such that $\left\|\sum_{k=1}^{n} T_{k}-T\right\| \rightarrow 0$ as $n \rightarrow \infty$. Let $\varepsilon>0$. For each $n$, we can find $\mathbf{y}_{n}=\left\{y_{n}^{k}\right\}_{k=1}^{\infty} \in l_{p}^{\mathrm{w}}(Y)$ and $S_{n} \in B\left(R\left(\mathbf{y}_{n}\right), X^{*}\right)$ with $T_{n}^{*}=S_{n} E_{\mathbf{y}_{n}}^{*}$ such that

$$
\left\|S_{n}\right\|<\left(\omega_{p}\left(T_{n}\right)+\varepsilon / 2^{n}\right)^{1 / p^{\prime}}, \quad\left\|\mathbf{y}_{n}\right\|_{p}^{\mathrm{w}}<\left(\omega_{p}\left(T_{n}\right)+\varepsilon / 2^{n}\right)^{1 / p} .
$$

Note that $\sum_{n=1}^{\infty}\left(\left\|\mathbf{y}_{n}\right\|_{p}^{\mathrm{w}}\right)^{p} \leq \sum_{n=1}^{\infty} \omega_{p}\left(T_{n}\right)+\varepsilon<\infty$ so that $\mathbf{y}=\left\{\mathbf{y}_{n}\right\} \in$ $l_{p}^{\mathrm{s}}\left(l_{p}^{\mathrm{w}}(Y)\right) \subset l_{p}^{\mathrm{w}}(Y)$. Next put $R=\overline{\bigoplus_{p}\left(R\left(\mathbf{y}_{n}\right)\right)}$. Then $R$ is a subspace of $l_{p}$ with $R=R(\mathbf{y})$. Now define $S: R \rightarrow X^{*}$ by

$$
S\left(\left\langle E_{\mathbf{y}_{n}}^{*}(f)\right\rangle\right)=\sum_{n=1}^{\infty} S_{n} E_{\mathbf{y}_{n}}^{*}(f), \quad f \in Y^{*} .
$$

Then

$$
\begin{aligned}
\|S\| & =\sup \left\{\left\|\sum_{n=1}^{\infty} S_{n} E_{\mathbf{y}_{n}}^{*}(f)\right\|:\left\|\left\langle E_{\mathbf{y}_{n}}^{*}(f)\right\rangle\right\|_{p} \leq 1\right\} \\
& \leq\left(\sum_{n=1}^{\infty}\left\|S_{n}\right\|^{p^{\prime}}\right)^{1 / p^{\prime}} \leq\left(\sum_{n=1}^{\infty} \omega_{p}\left(T_{n}\right)+\varepsilon\right)^{1 / p^{\prime}}<\infty .
\end{aligned}
$$

Thus $S \in B\left(R(\mathbf{y}), X^{*}\right)$. Moreover, $T^{*}(f)=\sum_{n=1}^{\infty} T_{n}^{*}(f)=\sum_{n=1}^{\infty} S_{n} E_{\mathbf{y}}^{*}(f)$ $=S E_{\mathbf{y}}^{*}(f)$ for all $f \in Y^{*}$. Thus $T^{*}=S E_{\mathbf{y}}^{*}$, and consequently $T \in W_{p}(X, Y)$. Also

$$
\omega_{p}(T) \leq\|S\| \cdot\|\mathbf{y}\|_{p}^{\mathrm{w}} \leq \sum_{n=1}^{\infty} \omega_{p}\left(T_{n}\right)+\varepsilon,
$$

so that $\omega_{p}(T) \leq \sum_{n=1}^{\infty} \omega_{p}\left(T_{n}\right)$, for $\varepsilon>0$ is arbitrary. The above construction 
yields, in general, for every $n \in \mathbb{N}$,

$$
\omega_{p}\left(T-\sum_{k=1}^{n} T_{k}\right) \leq \sum_{k=n+1}^{\infty} \omega_{p}\left(T_{k}\right) .
$$

Thus $\sum_{n=1}^{\infty} T_{n} \rightarrow T$ in the $\omega_{p}$-norm. In particular, if we take $T_{n}=0$ for $n>2$, we obtain $\omega_{p}\left(T_{1}+T_{2}\right) \leq \omega_{p}\left(T_{1}\right)+\omega_{p}\left(T_{2}\right)$.

Summing up, we conclude that $\left(W_{p}(X, Y), \omega_{p}\right)$ is a Banach operator ideal.

Along the lines of the above proof we can also obtain the following result.

TheOREM 4.2. $\left(K_{p}(X, Y), \kappa_{p}\right)$ is a Banach operator ideal.

Proposition 4.3. Let $1 \leq p<q \leq \infty$. Then $K_{p}(X, Y) \subset K_{q}(X, Y)$ for all Banach spaces $X$ and $Y$. Also, $\kappa_{q}(T) \leq \kappa_{p}(T)$ for any $T \in K_{p}(X, Y)$.

Proof. Let $T \in K_{p}(X, Y)$. Find $\mathbf{y}=\left\{y_{n}\right\} \subset \operatorname{Ball}(X)$ and $\boldsymbol{\lambda}=\left\langle\lambda_{n}\right\rangle \in l_{p}$ such that $T(\operatorname{Ball}(X)) \subset E_{\mathbf{y}} M_{\boldsymbol{\lambda}}\left(\operatorname{Ball}\left(l_{p^{\prime}}\right)\right)$. Put $\mu_{n}=\left|\lambda_{n}\right|^{p / q-1} \lambda_{n}$ (if $\lambda_{n} \neq 0$, and $\mu_{n}=0$ otherwise). Then $\sum_{n=1}^{\infty}\left|\mu_{n}\right|^{q}=\sum_{n=1}^{\infty}\left|\lambda_{n}\right|^{p}<\infty$, so that $\boldsymbol{\mu}=\left\langle\mu_{n}\right\rangle \in l_{q}$. Next, put $\beta_{n}=\left|\lambda_{n}\right|^{1-p / q}$ and $\boldsymbol{\beta}=\left\langle\beta_{n}\right\rangle$. Then $\boldsymbol{\beta} \in$ $l_{p q /(p-q)}$ and $M_{\boldsymbol{\lambda}}=M_{\boldsymbol{\mu}} M_{\boldsymbol{\beta}}$. Also, $M_{\boldsymbol{\beta}}\left(\operatorname{Ball}\left(l_{p^{\prime}}\right)\right) \subset\|\boldsymbol{\beta}\|_{p q /(q-p)} \operatorname{Ball}\left(l_{q^{\prime}}\right)=$ $\|\lambda\|_{p}^{1-p / q} \operatorname{Ball}\left(l_{q^{\prime}}\right)$. Thus

$$
T(\operatorname{Ball}(X)) \subset\|\lambda\|_{p}^{1-p / q} E_{\mathbf{y}} M_{\boldsymbol{\lambda}}\left(\operatorname{Ball}\left(l_{q^{\prime}}\right)\right),
$$

so that $T \in K_{q}(X, Y)$. Further, since $\|\boldsymbol{\lambda}\|_{p}^{1-p / q}\|\boldsymbol{\mu}\|_{q}=\|\boldsymbol{\lambda}\|_{p}$, we conclude that $\kappa_{q}(T) \leq \kappa_{p}(T)$.

5. Position of $\left(K_{p}, \kappa_{p}\right)$ in operator ideals. We note, by abuse of language, that at the " $p$-level" $(p \geq 1)$, the operator ideal of all $p$-summing operators is the largest and that of $p$-nuclear operators is the smallest. In this section we show that the dual of $\left(K_{p}, \kappa_{p}\right)$ lies between the above-mentioned two operator ideals. To this end we recall the following definitions.

Definition 5.1. Let $p \geq 1$ and let $X$ and $Y$ be Banach spaces. An operator $T \in B(X, Y)$ is said to be $p$-(absolutely) summing if $\left\{T x_{n}\right\} \in l_{p}^{\mathrm{s}}(Y)$ for all $\left\{x_{n}\right\} \in l_{p}^{\mathrm{w}}(X)$.

The set $\Pi_{p}(X, Y)$ of all $p$-summing operators forms a Banach space with the norm

$$
\pi_{p}(T)=\sup \left\{\left\|\left\{T x_{n}\right\}\right\|_{p}^{\mathrm{s}}:\left\|\left\{x_{n}\right\}\right\|_{p}^{\mathrm{w}} \leq 1\right\} .
$$

We note that $\left(\Pi_{p}, \pi_{p}\right)$ is an operator ideal.

Pietsch Factorization Theorem [7]. Let $X$ and $Y$ be Banach spaces and $p \geq 1$. Then $T \in \Pi_{p}(X, Y)$ if and only if there is a probability measure $\mu$ on $\operatorname{Ball}\left(X^{*}\right)$ and an operator $\widehat{T} \in B\left(X_{p}, Y\right)$ such that $T=\widehat{T} j_{p}^{X} i$. Here $i$ is the natural inclusion map from $X$ into $C\left(\operatorname{Ball}\left(X^{*}\right)\right), j_{p}^{X}=\left.j_{p}\right|_{i(X)}$ where 
$j_{p}$ is the canonical embedding of $C\left(\operatorname{Ball}\left(X^{*}\right)\right)$ into $L_{p}(\mu)$ and $X_{p}=\overline{j_{p} i(X)}$ is a closed subspace of $L_{p}(\mu)$. Moreover, we have $\|\widehat{T}\|=\pi_{p}(T)$.

Definition 5.2. For Banach spaces $X$ and $Y$ and $p \geq 1$, an operator $T \in B(X, Y)$ is said to be $p$-nuclear if there are $A \in B\left(l_{p}, Y\right), B \in B\left(X, c_{0}\right)$ and $\boldsymbol{\lambda} \in l_{p}$ such that $T=A M_{\lambda} B$.

The set $N_{p}(X, Y)$ of all $p$-nuclear operators in $B(X, Y)$ forms a Banach space with the norm

$$
\nu_{p}(T)=\inf \left\{\|A\| \cdot\|B\| \cdot\|\boldsymbol{\lambda}\|_{p}\right\},
$$

where the infimum is taken over all $p$-nuclear factorizations $T=A M_{\lambda} B$ of $T$. We again note that $\left(N_{p}, \nu_{p}\right)$ is an operator ideal.

Let $(A, \alpha)$ be a Banach operator ideal for Banach spaces $X$ and $Y$. Put

$$
A^{\mathrm{d}}(X, Y)=\left\{T \in B(X, Y): T^{*} \in A\left(Y^{*}, X^{*}\right)\right\} .
$$

For $T \in A^{\mathrm{d}}(X, Y)$, write $\alpha^{\mathrm{d}}(T)=\alpha\left(T^{*}\right)$. Then $\left(A^{\mathrm{d}}, \alpha^{\mathrm{d}}\right)$ is again an operator ideal and is called the dual ideal of $(A, \alpha)$.

If we compare the factorization of a $p$-summing operator, of a $p$-nuclear operator and that of the adjoint of a $p$-compact operator, we obtain the following result.

Proposition 5.3. Let $X$ and $Y$ be Banach spaces and $p \geq 1$. Then

(a) $N_{p}(X, Y) \subset K_{p}^{\mathrm{d}}(X, Y)$.

(b) $K_{p}(X, Y) \subset \Pi_{p}^{\mathrm{d}}(X, Y)$.

(c) $K_{p}^{\mathrm{d}}(X, Y) \subset \Pi_{p}(X, Y)$.

REMARK. (1) If $Y$ is reflexive, then it may be shown that $N_{p}^{\mathrm{d}}(X, Y) \subset$ $K_{p}(X, Y)$. However, we have not been able to prove or disprove it in general.

(2) In connection with the inclusions proved in the above proposition we can further show that the corresponding norms also have the desirable behaviour as follows:

(a) If $T \in N_{p}(X, Y)$, then $\nu_{p}(T) \geq \kappa_{p}^{\mathrm{d}}(T)$.

(b) If $T \in K_{p}(X, Y)$, then $\kappa_{p}(T) \geq \pi_{p}^{\mathrm{d}}(T)$.

(c) If $T \in K_{p}^{\mathrm{d}}(X, Y)$, then $\kappa_{p}^{\mathrm{d}}(T) \geq \pi_{p}(T)$.

Before we pass to some special cases concerning these operator ideals, we prove the following interesting result.

Proposition 5.4. For all $p \geq 1, p$-summing operators map weakly $p$ compact sets to $p$-compact sets. Moreover, for Banach spaces $X, Y$ and $Z$, we have

$$
\Pi_{p}(Y, Z) W_{p}(X, Y) \subset K_{p}(X, Z) .
$$

Proof. Let $T \in \Pi_{p}(X, Y)$ and let $C$ be a weakly $p$-compact set in $X$. Then there is an $\mathbf{x}=\left\{x_{n}\right\} \in l_{p}^{\mathrm{w}}(X)$ such that $C \subset \overline{E_{\mathbf{x}}\left(\operatorname{Ball}\left(l_{p^{\prime}}\right)\right)}$. Put 
$\mathbf{y}=\left\{T x_{n}\right\}$. Then $\mathbf{y} \in l_{p}^{\mathrm{s}}(Y)$ for $T \in \Pi_{p}(X, Y)$. Thus $T(C) \subset \overline{E_{\mathbf{y}}\left(\operatorname{Ball}\left(l_{p^{\prime}}\right)\right)}$ so that $T(C)$ is a $p$-compact set in $Y$.

Next, let $S \in \Pi_{p}(Y, Z)$ and $T \in W_{p}(X, Y)$. Then there is a $\mathbf{y}=\left\{y_{n}\right\} \in$ $l_{p}^{\mathrm{w}}(Y)$ such that $T(\operatorname{Ball}(X)) \subset \overline{E_{\mathbf{y}}\left(\operatorname{Ball}\left(l_{p^{\prime}}\right)\right)}$. Since $S \in \Pi_{p}(Y, Z)$, we get $\mathbf{z}=$ $\left\{S y_{n}\right\} \in l_{p}^{\mathrm{s}}(Z)$ so that $S T(\operatorname{Ball}(X)) \subset \overline{E_{\mathbf{z}}\left(\operatorname{Ball}\left(l_{p^{\prime}}\right)\right)}$ and $S T \in K_{p}(X, Z)$. Finally

$$
\kappa_{p}(S T) \leq\|\mathbf{z}\|_{p}^{\mathrm{S}} \leq \pi_{p}(S)\|\mathbf{y}\|_{p}^{\mathrm{w}} .
$$

Taking infimum over $\mathbf{y}$ we conclude that $\kappa_{p}(S T) \leq \pi_{p}(S) \omega_{p}(T)$.

REMARK. Recall that completely continuous operators map weakly compact sets into compact sets. Thus $p$-summing operators may be thought of as being " $p$-completely continuous" for $p \geq 1$.

Now we consider some special cases of the operator ideals discussed.

Proposition 5.5. Let $X$ be any Banach space. Then

(a) $\Pi_{p}\left(l_{p^{\prime}}, X\right) \subset E_{p}(X) \subset N_{p}^{\mathrm{d}}\left(l_{p^{\prime}}, X\right), p \geq 1$.

(b) $\Pi_{p}^{\mathrm{d}}\left(X, l_{p}\right) \subset E_{p}^{\mathrm{d}}(X) \subset N_{p}\left(X, l_{p}\right), p>1$.

Here $E_{p}^{\mathrm{d}}(X)=\left\{\left(E_{\mathbf{x}^{*}}\right)_{*}: \mathbf{x}^{*} \in l_{p}^{\mathrm{s}}\left(X^{*}\right)\right\}$, where $\left(E_{\mathbf{x}^{*}}\right)_{*}$ denotes the preadjoint of $E_{\mathbf{x}^{*}}$.

Proof. (a) Let $p \geq 1$ and $T \in \Pi_{p}\left(l_{p^{\prime}}, X\right)$. Since $\left\{e_{n}\right\} \in l_{p}^{\mathrm{w}}\left(l_{p^{\prime}}\right)$, we have $\left\{T e_{n}\right\} \in l_{p}^{\mathrm{s}}(X)$. Put $T e_{n}=x_{n}$ for all $n$. Then $T=E_{\mathbf{x}}$, so that $\Pi\left(l_{p^{\prime}}, X\right) \subset$ $E_{p}(X)$. Next, let $\mathbf{x} \in l_{p}^{\mathrm{s}}(X)$. Then $\left(E_{\mathbf{x}}\right)^{*}=I M_{\boldsymbol{\lambda}}\left(E_{\overline{\mathbf{x}}}\right)_{*}$, where $\boldsymbol{\lambda}=\left\langle\left\|x_{n}\right\|\right\rangle \in$ $l_{p}$ and $\overline{\mathbf{x}}=\left\{\bar{x}_{n}\right\}$ with $\bar{x}_{n}=\left\|x_{n}\right\|^{-1} x_{n}$ for all $n$. Thus $\left(E_{\mathbf{x}}\right)^{*} \in N_{p}\left(X^{*}, l_{p}\right)$ so that $E_{p}(X) \subset N_{p}^{\mathrm{d}}\left(l_{p^{\prime}}, X\right)$.

(b) Let $p>1$. If $T \in \Pi_{p}^{\mathrm{d}}\left(X, l_{p}\right)$, then $T^{*} \in \Pi_{p}\left(l_{p^{\prime}}, X^{*}\right)$. Thus as in (a), there is an $\mathbf{x}^{*} \in l_{p}^{\mathrm{s}}\left(X^{*}\right)$ such that $T^{*}=E_{\mathbf{x}^{*}}$ and it follows that $\Pi_{p}^{\mathrm{d}}\left(X, l_{p}\right) \subset$ $E_{p}^{\mathrm{d}}(X)$. Finally, let $\mathbf{x}^{*} \in l_{p}^{\mathrm{s}}\left(X^{*}\right)$. Then trivially, $\left(E_{\mathbf{x}^{*}}\right)_{*} \in N_{p}\left(X, l_{p}\right)$. Thus $E_{p}^{\mathrm{d}}(X) \subset N_{p}\left(X, l_{p}\right)$.

Remark. (i) Let $T \in \Pi_{p}\left(l_{p^{\prime}}, X\right)$ and find $\mathbf{x} \in l_{p}^{\mathrm{s}}(X)$ such that $T=E_{\mathbf{x}}$. Then it is easy to see that $\pi_{p}(T) \geq\|\mathbf{x}\|_{p}^{\mathrm{s}} \geq \nu_{p}^{\mathrm{d}}(T)$.

(ii) Again if $T \in \Pi_{p}^{\mathrm{d}}\left(X, l_{p}\right)$ and if $T=\left(E_{\mathbf{x}^{*}}\right)_{*}$ for some $\mathbf{x}^{*} \in l_{p}^{\mathrm{s}}\left(X^{*}\right)$, we can show that $\pi_{p}^{\mathrm{d}}(T) \geq\left\|\mathrm{x}^{*}\right\|_{p}^{\mathrm{s}} \geq \nu(T)$.

(iii) It follows from the definition that $E_{p}(X) \subset K_{p}\left(l_{p^{\prime}}, X\right)$ for $p \geq 1$.

(iv) It follows from the above propositions that

(a) $N_{p}\left(l_{p^{\prime}}, X\right) \subset K_{p}^{\mathrm{d}}\left(l_{p^{\prime}}, X\right) \subset \Pi_{p}\left(l_{p^{\prime}}, X\right) \subset E_{p}(X) \subset N_{p}^{\mathrm{d}}\left(l_{p^{\prime}}, X\right)$ for $p \geq 1$.

(b) $N_{p}^{\mathrm{d}}\left(X, l_{p}\right) \subset K_{p}\left(X, l_{p}\right) \subset \Pi_{p}^{\mathrm{d}}\left(X, l_{p}\right) \subset E_{p}^{\mathrm{d}}(X) \subset N_{p}\left(X, l_{p}\right) \subset$ $K_{p}^{\mathrm{d}}\left(X, l_{p}\right) \subset \Pi_{p}\left(X, l_{p}\right)$ for $p>1$.

(c) $N_{p}^{\mathrm{d}}\left(l_{p^{\prime}}, X\right) \subset K_{p}\left(l_{p^{\prime}}, X\right) \subset \Pi_{p}^{\mathrm{d}}\left(l_{p^{\prime}}, X\right)$ for $p>1$. 
(v) Putting $X=l_{p}$ in (iv)(a) and $X=l_{p^{\prime}}$ in (iv)(b), we obtain $N_{p}\left(l_{p^{\prime}}, l_{p}\right)$ $=N_{p}^{\mathrm{d}}\left(l_{p^{\prime}}, l_{p}\right)=K_{p}\left(l_{p^{\prime}}, l_{p}\right)=K_{p}^{\mathrm{d}}\left(l_{p^{\prime}}, l_{p}\right)=\Pi_{p}\left(l_{p^{\prime}}, l_{p}\right)=\Pi_{p}^{\mathrm{d}}\left(l_{p^{\prime}}, l_{p}\right)=E_{p}\left(l_{p}\right)$ $=E_{p}^{\mathrm{d}}\left(l_{p^{\prime}}\right)$ (isometrically) for $p>1$.

6. Approximation on $p$-compact sets. Following Grothendieck [5], a Banach space $X$ is said to have the approximation property (for short, a.p.) if the identity operator can be approximated uniformly on compact sets by finite rank operators. Equivalently, a Banach space has the a.p. if and only if the identity operator is in the closure of the finite rank operators in the locally convex topology of uniform convergence on compact sets. The question whether every Banach space has the a.p., raised in 1956 by Grothendieck himself, was solved by Enflo [4] in 1973 by producing a separable Banach space that fails the a.p. This was followed by several authors giving simpler constructions of Banach spaces without the a.p. For instance, Davie $[1,2]$ gave a probabilistic construction. A comprehensive exposition of this problem and its solutions can be found in the book of Lindenstrauss and Tzafriri [6].

From this section onwards, we study weaker forms of approximating the identity operator by finite rank operators. We start with the following definition.

Definition 6.1. A Banach space $X$ is said to have the $p$-approximation property (for short, $p$-a.p.), for $p \geq 1$, if for every $p$-compact set $K$ in $X$ and every $\varepsilon>0$, there is a finite rank operator $T \in B(X)$ such that $\|T x-x\|<\varepsilon$ for all $x \in K$.

Let $X$ and $Y$ be Banach spaces and $1 \leq p \leq \infty$. Let $\tau_{p}$ denote the topology in $B(X, Y)$ of uniform convergence on $p$-compact sets in $X$. Since finite sets are $p$-compact, $\tau_{p}$ is a locally convex topology generated by seminorms of the form

$$
\|T\|_{K}=\sup \{\|T x\|: x \in K\}, \quad T \in B(X, Y),
$$

where $K$ varies over all $p$-compact sets in $X$. Let $\mathbf{x}=\left\{x_{n}\right\} \in l_{p}^{\mathrm{s}}(X)$. Then $K=E_{\mathbf{x}}\left(\operatorname{Ball}\left(l_{p^{\prime}}\right)\right)$ is a $p$-compact set in $X$ and we have

$$
\begin{aligned}
\|T\|_{K} & =\sup \left\{\|T y\|: y \in E_{\mathbf{x}}\left(\operatorname{Ball}\left(l_{p^{\prime}}\right)\right)\right\} \\
& =\left\|E_{\left\langle T x_{n}\right\rangle}\right\|=\left\|\left\{T x_{n}\right\}\right\|_{p}^{\mathrm{w}}, \quad T \in B(X, Y) .
\end{aligned}
$$

Write $\left\|\left\langle T x_{n}\right\rangle\right\|_{p}^{\mathrm{w}}=\|T\|_{\mathbf{x}}$ for all $\mathbf{x}=\left\{x_{n}\right\} \in l_{p}^{\mathrm{s}}(X)$ and $T \in B(X, Y)$. Then the family $\left\{\|\cdot\|_{\mathbf{x}}: \mathbf{x} \in l_{p}^{\mathrm{s}}(X)\right\}$ of seminorms generates the topology $\tau_{p}$. Moreover, we note that $X$ has the $p$-a.p. if and only if given $\mathbf{x} \in l_{p}^{\mathrm{s}}(X)$ and $\varepsilon>0$, there is an $S \in F(X)$ such that $\|S-I\|_{\mathbf{x}} \leq \varepsilon$.

The following result is immediate. 
Proposition 6.2. Let $1 \leq p \leq \infty$. Then for a Banach space $X$ the following statements are equivalent:

(a) $X$ has the p-approximation property.

(b) For any Banach space $Y$ the set $F(Y, X)$ (respectively, $F(X, Y)$ ) of finite rank operators is $\tau_{p}$-dense in $B(Y, X)$ (respectively, $B(X, Y)$ ).

The classical approximation property for a Banach space $X$ is equivalent to the density of the finite rank operators in the ideal $K(Y, X)$ of compact operators for every Banach space $Y$ in the operator norm. For $p$-a.p. we have the following surprising result.

Theorem 6.3. Let $1 \leq p \leq \infty$. Then a Banach space $X$ has the $p$ approximation property if and only if for every Banach space $Y, F(Y, X)$ is $\omega_{p}$-dense in $K_{p}(Y, X)$.

Proof. First assume that $X$ has the $p$-a.p. Let $Y$ be a Banach space, $T \in K_{p}(Y, X)$ and $\varepsilon>0$. Then there is an $\mathbf{x} \in l_{p}^{\mathrm{s}}(X)$ such that $T(\operatorname{Ball}(Y))$ $\subset E_{\mathbf{x}}\left(\operatorname{Ball}\left(l_{p^{\prime}}\right)\right)$. Since $X$ has the $p$-a.p., there is an $S \in F(X)$ such that $\|S-I\|_{\mathbf{x}} \leq \varepsilon$. Put $S_{1}=S T$. Then $S_{1} \in F(Y, X)$ and we have

$$
\begin{aligned}
\left(S_{1}-T\right)(\operatorname{Ball}(Y)) & =(S-I) T(\operatorname{Ball}(Y)) \subset(S-I) E_{\mathbf{x}}\left(\operatorname{Ball}\left(l_{p^{\prime}}\right)\right) \\
& =E_{\left\{S x_{n}-x_{n}\right\}}\left(\operatorname{Ball}\left(l_{p^{\prime}}\right)\right) .
\end{aligned}
$$

Thus $\omega_{p}\left(S_{1}-T\right) \leq\left\|\left\{S x_{n}-x_{n}\right\}\right\|_{p}^{\mathrm{w}}=\|S-I\|_{\mathbf{x}} \leq \varepsilon$ so that $F(Y, X)$ is $\omega_{p}$-dense in $K_{p}(Y, X)$.

For the converse, suppose that for any Banach space $Y, F(Y, X)$ be $\omega_{p}$-dense in $K_{p}(Y, X)$. Then the proof that $X$ has the $p$-a.p. is a variation of the proof of the implication $(\mathrm{v}) \Rightarrow(\mathrm{i})$ in [6, Theorem 1.e.4]. We only need to mention the following. Let $\mathbf{x}=\left\{x_{n}\right\} \in l_{p}^{\mathrm{s}}(X)$ and $K=E_{\mathbf{x}}\left(\operatorname{Ball}\left(l_{p^{\prime}}\right)\right)$. We can choose $t_{n}>0$ for all $n$ such that $t_{n} \rightarrow \infty$ as $n \rightarrow \infty$ and $\sum_{n=1}^{\infty} t_{n}^{p}\left\|x_{n}\right\|^{p}<\infty$. Put $z_{n}=t_{n} x_{n}$ and $U=E_{\mathbf{z}}\left(\operatorname{Ball}\left(l_{p^{\prime}}\right)\right)$, where $\mathbf{z}=\left\{z_{n}\right\} \in l_{p}^{\mathrm{s}}(X)$. Then $U$ is a compact convex set symmetric with respect to origin, which is also a $p$-compact set.

REMARK. In the above result one expects that $\omega_{p}$ could be replaced by $\kappa_{p}$. In this regard it would be desirable to introduce some other type of approximation property of Banach spaces. However, our definition (of the $p$-approximation property) exhibits the following beautiful behavior in Banach spaces. (Nevertheless, we consider two other notions of $p$-approximation properties, one due to P. D. Saphar and the other due to O. I. Reĭnov, in the next section.)

THEOREM 6.4. Every Banach space has the 2-approximation property (and thus the p-approximation property if $1 \leq p \leq 2$ ).

In order to prove this result we need the following lemma. 
Lemma 6.5. Let $X$ be a Banach space, $H$ a Hilbert space, $K$ a compact set in $H$ and $S \in B(H, X)$. Then the identity operator on $X$ can be approximated uniformly on $S(K)$ by finite rank operators.

Proof. Without loss of generality, we may assume that $S$ has zero null space. Indeed, we can replace $H$ by $H^{\prime}=H / N(S), K$ by $K^{\prime}=\{x+N(S)$ : $x \in K\}$ and $S$ by the induced map $S^{\prime} \in B\left(H^{\prime}, X\right)$.

Now, fix $\varepsilon>0$. Let $P$ be a finite rank operator on $H$ with $\|P x-x\|<\varepsilon$ for all $x \in K$. Since $N(S)=\{0\}$, the set of functionals $\varphi$ on $X$ defined by $\varphi(x)=\psi(S x)$ where $\psi \in X^{*}$, is dense in $H^{*}$; so we can find a finite rank operator $T \in B(X, H)$ such that $\|P-T S\|<\varepsilon$ and it follows that

$$
\|T S x-x\|<(1+M) \varepsilon \quad \text { for all } x \in K,
$$

where $M=\sup \{\|x\|: x \in K\}$. Consequently, $\|S T y-y\|<(1+M)\|S\| \varepsilon$ for all $y \in S(K)$.

Proof of Theorem 6.4. Let $X$ be a Banach space, $\mathbf{x}=\left\{x_{n}\right\}=l_{2}^{\mathrm{s}}(X)$ and $\varepsilon>0$. We can find $\boldsymbol{\lambda}=\left\langle\lambda_{n}\right\rangle \in c_{0}$ and $\mathbf{z}=\left\{z_{n}\right\} \in l_{2}^{\mathrm{s}}(X)$ such that $\lambda_{n}>0$ and $x_{n}=\lambda_{n} z_{n}$ for all $n$. Consider $E_{\mathbf{z}} \in E_{2}(X) \subset B\left(l_{2}, X\right)$ and put $K_{0}=\overline{M_{\lambda}\left(\operatorname{Ball}\left(l_{2}\right)\right)}$. Then $K_{0}$ is a compact set in $l_{2}$. Thus by Lemma 6.5 , there is an $S \in F(X)$ such that $\|(S-I) y\| \leq \varepsilon$ for all $y \in E_{\mathbf{z}}\left(\overline{M_{\lambda}\left(\operatorname{Ball}\left(l_{2}\right)\right)}\right)=$ $\overline{E_{\mathbf{x}}\left(\operatorname{Ball}\left(l_{2}\right)\right)}$. It follows that $\|S-I\|_{\mathbf{x}} \leq \varepsilon$, so that $X$ has the 2-a.p.

REMARK. The above result is optimal in the sense that for every $p>2$ there is a Banach space that fails the $p$-a.p. To this end we need the following probabilistic construction due to Davie $[1,2]$.

Lemma 6.6. There exists an infinite matrix $A=\left(a_{n m}\right)_{n, m=1}^{\infty}$ of scalars in which for each $n, a_{n m}=0$ for all but finitely many indices $m$, such that

(i) $\sum_{n=1}^{\infty}\left(\max _{1 \leq m<\infty}\left|a_{n m}\right|\right)^{r}<\infty$ for every $r>2 / 3$,

(ii) $A^{2}=0$,

(iii) $\operatorname{trace} A \neq 0$.

It is interesting to note that Grothendieck [5, Remark I.14] observed that if an infinite matrix $A=\left(a_{n m}\right)_{n, m=1}^{\infty}$ satisfies $\lim _{m \rightarrow \infty} a_{n m}=0$ for all $n$, $\sum_{n=1}^{\infty}\left(\max _{1 \leq m<\infty}\left|a_{n m}\right|\right)^{2 / 3}<\infty$ and $A^{2}=0$, then trace $A=0$. A proof of this is indicated in [6, Remark 1, p. 90]. Another proof was obtained by Reĭnov [10].

Theorem 6.7. Let $p>2$. Then there is a Banach space that fails the p-a.p.

Proof. Put $r=p(p+1)^{-1}$ so that $r>2 / 3$. In Lemma 6.6 put $a_{n}=$ $\max _{1 \leq m<\infty}\left|a_{n m}\right|$ for all $n$. Then $\left\langle a_{n}\right\rangle \in l_{r}$. Thus we can find $\left\langle\lambda_{n}\right\rangle \in l_{1}$ and $\left\langle b_{n}\right\rangle \in l_{p}$ such that $\lambda_{n}>0$ and $a_{n}=\lambda_{n} b_{n}$ for all $n$. Put $b_{n m}=\lambda_{n}^{-1} a_{n m}$ for all $n, m \in \mathbb{N}$. Then for each $n, b_{n m}=0$ for all but finitely many indices $m$. 
Put $x_{n}=\left\langle b_{n m}\right\rangle_{m=1}^{\infty}$. Let $E$ be the closed linear span of $\left\{x_{n}\right\}$ in $c_{0}$ and let $f_{n}=\left.\lambda_{n} e_{n}\right|_{E}$ for all $n$, where $\left\{e_{n}\right\}$ is the standard unit vector basis of $l_{1}$. Then $\left\{x_{n}\right\} \in l_{p}(E)$ and $\left\{f_{n}\right\} \in l_{1}\left(E^{*}\right)$. Note that

$$
\sum_{n=1}^{\infty} f_{n}\left(x_{m}\right) x_{n}=\left\langle\sum_{n=1}^{\infty} \lambda_{n} b_{m n} b_{n k}\right\rangle_{k}=\lambda_{m}^{-1}\left\langle\sum_{n=1}^{\infty} a_{m n} a_{n k}\right\rangle_{k}=0
$$

for all $m$, so that $\sum_{n=1}^{\infty} f_{n}(x) x_{n}=0$ for all $x \in E$. However, $\sum_{n=1}^{\infty} f_{n}\left(x_{n}\right)=$ $\sum_{n=1}^{\infty} \lambda_{n} b_{n n}=\sum_{n=1}^{\infty} a_{n n} \neq 0$. Now, it follows from the next result that $E$ fails the $p$-a.p.

Proposition 6.8. Let $X$ be a Banach space and let $1 \leq p \leq \infty$. If $X$ has the p-approximation property then for every choice of $\left\{x_{n}\right\} \in l_{p}^{\mathrm{s}}(X)$ and $\left\{f_{n}\right\} \in l_{1}^{\mathrm{s}}\left(X^{*}\right)$ with $\sum_{n=1}^{\infty} f_{n}(x) x_{n}=0$ for all $x \in X$, we have $\sum_{n=1}^{\infty} f_{n}\left(x_{n}\right)$ $=0$.

Proof. Let $X$ have the $p$-a.p. Let $\left\{x_{n}\right\} \in l_{p}^{\mathrm{s}}(X)$ and $\left\{f_{n}\right\} \in l_{1}^{\mathrm{s}}\left(X^{*}\right)$ be such that $\sum_{n=1}^{\infty} f_{n}(x) x_{n}=0$ for all $x \in X$. Then by linearity $\sum_{n=1}^{\infty} f_{n}\left(S x_{n}\right)$ $=0$ for all $S \in F(X)$. Now for each $\varepsilon>0$ there is an $S \in F(X)$ such that $\left\|\left\{S x_{n}-x_{n}\right\}\right\|_{p}^{\mathrm{w}} \leq \varepsilon$. Thus

$$
\begin{aligned}
\left|\sum_{n=1}^{\infty} f_{n}\left(x_{n}\right)\right| & =\left|\sum_{n=1}^{\infty} f_{n}\left(S x_{n}-x_{n}\right)\right| \leq\left\|\left\{f_{n}\right\}\right\|_{1}^{\mathrm{s}}\left\|\left\{S x_{n}-x_{n}\right\}\right\|_{\infty} \\
& \leq\left\|\left\{f_{n}\right\}\right\|_{1}^{\mathrm{s}}\left\|\left\{S x_{n}-x_{n}\right\}\right\|_{p}^{\mathrm{w}} \leq \varepsilon\left\|\left\{f_{n}\right\}\right\|_{1}^{\mathrm{s}} .
\end{aligned}
$$

Since $\varepsilon>0$ is arbitrary, we obtain $\sum_{n=1}^{\infty} f_{n}\left(x_{n}\right)=0$.

\section{Connections with $p$-approximation properties of Saphar and}

Reinnov. Grothendieck [5] proved that a Banach space $X$ has the a.p. if and only if for every Banach space $Y$ (equivalently, for $Y=X$ ), the natural map from $Y^{*} \widetilde{\otimes}_{\pi} X$ into $B(Y, X)$ is one-to-one. Weaker forms of the a.p. were defined by restricting the above maps to certain subspaces of the projective tensor product. For instance, let $g_{p}$ for $p \geq 1$ be the projective tensor norm of Saphar [11] and let $Y^{*} \widetilde{\otimes}_{g_{p}} X$ be the completion of the tensor product of $Y^{*}$ and $X$ under this norm. If $1 / p+1 / p^{\prime}=1$, then $\left(Y^{*} \widetilde{\otimes}_{g_{p}} X\right)^{*}=\Pi_{p^{\prime}}\left(X, Y^{* *}\right)$. Now, Saphar's $p$-approximation property (for short, Saphar's $p$-a.p.) defined in [12] is equivalent to the following: A Banach space $X$ has Saphar's p-a.p. if and only if the natural map from $Y^{*} \widetilde{\otimes}_{g_{p^{\prime}}} X$ into $B(Y, X)$ is one-to-one. In the same direction Reĭnov [9] also studied a $p$-approximation property (for short, Reऍnov's $p$-a.p.) for $0<p<\infty$. Consider the subspace $\left(Y^{*} \widetilde{\otimes}_{\pi} X\right)_{p}$ of $Y^{*} \widetilde{\otimes}_{\pi} X$ consisting of elements $\sum_{n=1}^{\infty} f_{n} \otimes x_{n}$ with $\left\{f_{n}\right\} \subset Y^{*}$ and $\left\{x_{n}\right\} \subset X$ satisfying $\sum_{n=1}^{\infty}\left(\left\|f_{n}\right\| \cdot\left\|x_{n}\right\|\right)^{p}<\infty$. Then a Banach space $X$ is said to have Reřnov's $p$-a.p. if for every Banach space $Y$, the natural map from $\left(Y^{*} \widetilde{\otimes}_{\pi} X\right)_{p}$ into $B(Y, X)$ is one-to-one. 
In Proposition 6.8 , we essentially used the fact that $\|\cdot\|_{\infty} \leq\|\cdot\|_{p}^{\mathrm{w}}$ [on $\left.l_{p}^{\mathrm{w}}(X)\left(\subset l_{\infty}(X)\right)\right]$. This fact motivates us to further weaken the definition of the $p$-approximation property.

Definition 7.1. Let $1 \leq p \leq q \leq \infty$. We say that a Banach space $X$ has the $(q, p)$-approximation property (for short, $(q, p)$-a.p.) if given $\left\{x_{n}\right\} \in$ $l_{p}^{\mathrm{s}}(X)\left(\left\{x_{n}\right\} \in c_{0}^{\mathrm{s}}(X)\right.$ if $\left.p=\infty\right)$ and $\varepsilon>0$ there is an $S \in F(X)$ such that $\left\|\left\{S x_{n}-x_{n}\right\}\right\|_{q}^{\mathrm{s}} \leq \varepsilon$.

Note. (1) $(\infty, \infty)$-a.p. $=$ a.p. $(=\infty$-a.p. $)$.

(2) p-a.p. $\Rightarrow(\infty, p)$-a.p.

(3) (q,p)-a.p. $\Rightarrow(t, s)$-a.p. if $1 \leq s \leq p \leq q \leq t \leq \infty$.

Let $X$ and $Y$ be Banach spaces and $1 \leq p \leq q \leq \infty$. For $\mathbf{x}=\left\{x_{n}\right\} \in$ $l_{p}^{\mathrm{s}}(X)\left(\mathbf{x} \in c_{0}^{\mathrm{s}}(X)\right.$ if $\left.p=\infty\right)$, we define

$$
\|T\|_{q, \mathbf{x}}=\left\|\left\{T x_{n}\right\}\right\|_{q}^{\mathrm{s}}, \quad T \in B(X, Y) .
$$

The family $\left\{\|\cdot\|_{q, \mathbf{x}}\right\}$ of seminorms defines a locally convex topology on $B(X, Y)$ which is denoted by $\tau_{q, p}$. It is routine to verify the following result.

Proposition 7.2. Let $1 \leq p \leq q \leq \infty$. Then for any Banach space $X$ the following statements are equivalent:

(a) $X$ has the $(q, p)$-approximation property.

(b) For any Banach space $Y, F(X, Y)$ (respectively, $F(Y, X))$ is $\tau_{q, p^{-}}$ dense in $B(X, Y)$ (respectively, $B(Y, X))$.

Lemma 7.3. Let $1 \leq p \leq q \leq \infty$ and let $X$ and $Y$ be Banach spaces. Then the $\tau_{q, p}$-continuous linear functionals on $B(X, Y)$ are precisely those of the form

$$
\phi(T)=\sum_{n=1}^{\infty} g_{n}\left(T x_{n}\right), \quad T \in B(X, Y),
$$

where $\left\{x_{n}\right\} \in l_{p}^{\mathrm{s}}(X)$ and $\left\{g_{n}\right\} \in l_{q^{\prime}}^{\mathrm{s}}\left(Y^{*}\right)$.

Proof. Let $\mathbf{x}=\left\{x_{n}\right\} \in l_{p}^{\mathrm{s}}(X)$ and $\left\{g_{n}\right\} \in l_{q^{\prime}}^{\mathrm{s}}\left(Y^{*}\right)$. Then

$$
\begin{aligned}
\sum_{n=1}^{\infty}\left|g_{n}\left(T x_{n}\right)\right| & \leq \sum_{n=1}^{\infty}\left\|g_{n}\right\| \cdot\left\|T x_{n}\right\| \leq\left\|\left\{g_{n}\right\}\right\|_{q^{\prime}}^{\mathrm{S}}\left\|\left\{T x_{n}\right\}\right\|_{q}^{\mathrm{s}} \\
& =\left\|\left\{g_{n}\right\}\right\|_{q^{\prime}}^{\mathrm{S}}\|T\|_{q, \mathbf{x}}, \quad T \in B(X, Y) .
\end{aligned}
$$

Thus $\phi$ given by $(\dagger)$ is $\tau_{q, p}$-continuous.

Conversely, let $\phi$ be a $\tau_{q, p}$-continuous linear functional on $B(X, Y)$. Then there is an $\mathbf{x}=\left\{x_{n}\right\} \in l_{p}^{\mathrm{s}}(X)$ and $M>0$ such that

$$
|\phi(T)| \leq M\|T\|_{q, \mathbf{x}}, \quad T \in B(X, Y) .
$$


Define $S_{\mathbf{x}}: B(X, Y) \rightarrow l_{q}^{\mathrm{s}}(Y)$ by $S_{\mathbf{x}}(T)=\left\{T x_{n}\right\}, T \in B(X, Y)$. Then $S_{\mathbf{x}} \in B\left(B(X, Y), l_{q}^{\mathrm{S}}(Y)\right)$ and consequently we obtain a bounded linear functional $\psi$ on $S_{\mathbf{x}}(B(X, Y)) \subset l_{q}^{\mathrm{s}}(Y)$ given by $\psi\left(S_{\mathbf{x}}(T)\right)=\phi(T), T \in B(X, Y)$. Extending $\psi$ to $l_{q}^{\mathrm{s}}(Y)$, by the Hahn-Banach theorem, we obtain $\left\{g_{n}\right\} \in$ $l_{q^{\prime}}^{\mathrm{s}}\left(Y^{*}\right) \cong\left(l_{q}^{\mathrm{s}}(Y)\right)^{*}$ with $\phi(T)=\psi\left(S_{\mathbf{x}}(T)\right)=\left\langle\left\{g_{n}\right\},\left\{T x_{n}\right\}\right\rangle=\sum_{n=1}^{\infty} g_{n}\left(T x_{n}\right)$ for all $T \in B(X, Y)$.

REMARK. A $\tau_{q, p}$-continuous linear functional on $B(X, Y)$ is given by (†) where $\left\{x_{n}\right\} \subset X,\left\{g_{n}\right\} \subset Y^{*}$ and $\sum_{n=1}^{\infty}\left(\left\|x_{n}\right\| \cdot\left\|g_{n}\right\|\right)^{s}<\infty$ where $1 / s=1 / p+1 / q^{\prime}$. Thus the $\tau_{q, p^{-}}$topological dual of $B(X, Y)$ is identical with $\tau_{\infty, r}$-topological dual where $1 / r=1 / p-1 / q$.

Theorem 7.4. Let $1 \leq p \leq q \leq \infty$. Then a Banach space $X$ has the $(q, p)$-approximation property if and only if for every choice of $\left\{x_{n}\right\} \in$ $l_{p}^{\mathrm{s}}$ and $\left\{f_{n}\right\} \in l_{q^{\prime}}^{\mathrm{s}}\left(X^{*}\right)$ with $\sum_{n=1}^{\infty} f_{n}(x) x_{n}=0$ for all $x \in X$, we have $\sum_{n=1}^{\infty} f_{n}\left(x_{n}\right)=0$.

Proof. Duplicating the proof of Proposition 6.8, we obtain the necessary part.

Conversely, assume that for $\left\{x_{n}\right\} \in l_{p}^{\mathrm{s}}(X)$ and $\left\{f_{n}\right\} \in l_{q^{\prime}}^{\mathrm{s}}\left(X^{*}\right)$ with $\sum_{n=1}^{\infty} f_{n}(x) x_{n}=0$ for all $x \in X$, we have $\sum_{n=1}^{\infty} f_{n}\left(x_{n}\right)=0$. We shall prove that $F(X)$ is $\tau_{q, p^{-}}$-dense in $B(X)$. Let $\phi$ be a $\tau_{p^{-}}$-continuous linear functional on $B(X)$ vanishing on $F(X)$. There are $\left\{x_{n}\right\} \in l_{p}^{\mathrm{s}}(X)$ and $\left\{f_{n}\right\} \in l_{q^{\prime}}\left(X^{*}\right)$ such that $\varphi(T)=\sum_{n=1}^{\infty} f_{n}\left(T x_{n}\right)$ for all $T \in B(X)$. Thus $\sum_{n=1}^{\infty} f_{n}\left(S x_{n}\right)=0$ if $S \in F(X)$. In particular,

$$
f\left(\sum_{n=1}^{\infty} f_{n}(x) x_{n}\right)=\sum_{n=1}^{\infty} f_{n}\left((f \otimes x) x_{n}\right)=0, \quad x \in X, f \in X^{*},
$$

so that $\sum_{n=1}^{\infty} f_{n}(x) x_{n}=0$ for all $x \in X$ and consequently $\sum_{n=1}^{\infty} f_{n}(x) T x_{n}=$ 0 for all $x \in X$ and $T \in B(X)$. Now it follows from the hypothesis that $\phi(T)=\sum_{n=1}^{\infty} f_{n}\left(T x_{n}\right)=0, T \in B(X)$, so that $F(X)$ is $\tau_{q, p}$-dense in $B(X)$. Hence by Proposition 7.2, $X$ has the $(q, p)$-a.p.

COROLlary 7.5. A Banach space $X$ has the $(q, p)$-approximation property if and only if it has the $(\infty, r)$-approximation property where $1 / r=$ $1 / p-1 / q$, if and only if it has Reinnov's $r(r+1)^{-1}$-approximation property.

The following theorem can be found in [9] (in Reunov's terminology).

THEOREM 7.6. Every Banach space has the $(\infty, 2)$-approximation property. For each $p>2$ there is a Banach space which fails the $(\infty, p)$-approximation property.

Proof. Since $p$-a.p. implies $(\infty, p)$-a.p., it follows from Theorem 6.4 that every Banach space has the $(\infty, 2)$-a.p. Now, for given $p>2$ the proof of Theorem 6.7 shows that the Banach space $E$ fails the $(\infty, p)$-a.p. 
Remark. Since a.p. $=(\infty, \infty)$-a.p., we see that a Banach space $X$ has the a.p. if and only if it has the $(p, p)$-a.p. for all $p \geq 1$. Duplicating the proof of Theorem 6.3, we conclude that if $X$ has the a.p., then for all $p \geq 1$, $F(Y, X)$ is $\kappa_{p}$-dense in $K_{p}(X, Y)$ for any Banach space $Y$ (we expect that the converse is true as well).

We propose to call $\tau_{q, p}$ the topology of $q$-convergence on $p$-compact sets. This notion may be further explored in another direction to meet the notion of Saphar's p-a.p.

Let $X$ and $Y$ be Banach spaces and $1 \leq p \leq q \leq \infty$. Let $\sigma_{q, p}$ denote the topology on $\Pi_{p}(X, Y)$ of $q$-convergence on weakly p-compact sets of $X$, which is defined as follows: Let $K$ be a weakly $p$-compact set in $X$ and $T \in \Pi_{p}(X, Y)$. Then there is an $\mathbf{x} \in l_{p}^{\mathrm{w}}(X)$ such that $K \subset E_{\mathbf{x}}\left(\operatorname{Ball}\left(l_{p^{\prime}}\right)\right)$, so that $T(K)$ is a $p$-compact set in $Y$. We define

$$
\|T\|_{K}^{\mathrm{w}}=\sup \{\|T x\|: x \in K\}, \quad T \in \Pi_{p}(X, Y) .
$$

Restricting to $K=E_{\mathbf{x}}\left(\operatorname{Ball}\left(l_{p^{\prime}}\right)\right)$ and $\mathbf{x} \in l_{p}^{\mathrm{w}}(X)$ as before, we may define $\|T\|_{\mathbf{x}}^{\mathrm{w}}=\|T\|_{K}^{\mathrm{w}}=\left\|\left\{T x_{n}\right\}\right\|_{p}^{\mathrm{w}}$, and may generalize this idea to obtain seminorms $\|\cdot\|_{q, \mathbf{x}}^{\mathrm{w}}$ on $\Pi_{p}(X, Y)$ where

$$
\|T\|_{q, \mathbf{x}}^{\mathrm{w}}=\left\|\left\{T x_{n}\right\}\right\|_{q}^{\mathrm{s}}, \quad T \in \Pi_{p}(X, Y) .
$$

The family $\left\{\|\cdot\|_{q, \mathbf{x}}^{\mathrm{w}}: \mathbf{x} \in l_{p}^{\mathrm{w}}(X)\right\}$ of seminorms determines the locally convex topology $\sigma_{q, p}$ on $\Pi_{p}(X, Y)$. It is now routine to verify that the $\sigma_{q, p^{-}}$ continuous linear functionals on $\Pi_{p}(X, Y)$ are precisely those of the form

$$
\phi(T)=\sum_{n=1}^{\infty} g_{n}\left(T x_{n}\right), \quad\left\{x_{n}\right\} \in l_{p}^{\mathrm{w}}(X),\left\{g_{n}\right\} \in l_{q^{\prime}}^{\mathrm{s}}\left(Y^{*}\right) .
$$

Finally, we introduce the following weak topology version of Definition 7.1.

Definition 7.7. Let $1 \leq p \leq q \leq \infty$. A Banach space is said to have the weak $(q, p)$-approximation property (for short, weak $(q, p)$-a.p.) if for every Banach space $Y, \mathbf{x}=\left\{x_{n}\right\} \in l_{p}^{\mathrm{w}}(X), \varepsilon>0$ and $T \in \Pi_{p}(X, Y)$ there is an $S \in F(X, Y)$ such that $\|S-T\|_{q, \mathbf{x}}^{\mathrm{w}} \leq \varepsilon$.

Note. (1) For $1 \leq s \leq p \leq q \leq t \leq \infty$, weak (q,p)-a.p. $\Rightarrow$ weak $(t, s)$-a.p.

(2) Weak $(p, p)$-a.p. = Saphar's $p$-a.p., for all $p \geq 1$ [12].

(3) It was also shown in [12] that every Banach space has Saphar's 2-a.p.

(4) Since a.p. $\Rightarrow$ Saphar's $p$-a.p., we conclude that a.p. $\Rightarrow$ weak $(q, p)$-a.p. for all $1 \leq p \leq q \leq \infty$.

Lastly, we record the following theorem for the sake of completeness.

Theorem 7.8. Let $X$ be a Banach space and $1 \leq p \leq q \leq \infty$. Then the following statements are equivalent: 
(a) $X$ has the weak (q,p)-approximation property.

(b) For every Banach space $Y, F(X, Y)$ is $\sigma_{q, p}$-dense in $B(X, Y)$.

(c) For every Banach space $Y$, and every choice of $\left\{x_{n}\right\} \in l_{p}^{\mathrm{w}}(X)$ and $\left\{g_{n}\right\} \in l_{q^{\prime}}^{\mathrm{s}}\left(Y^{*}\right)$ with $\sum_{n=1}^{\infty} f\left(x_{n}\right) g_{n}=0$ for all $f \in X^{*}$, we have $\sum_{n=1}^{\infty} g_{n}\left(T x_{n}\right)=0$ for all $T \in \Pi_{p}(X, Y)$.

Acknowledgements. The authors are indebted to Eve Oja for indicating the connection between the $p$-approximation property considered in this paper and that studied by O. I. Reĭnov.

\section{References}

[1] A. M. Davie, The approximation problem for Banach spaces, Bull. London Math. Soc. 5 (1973), 261-266.

[2] - The Banach approximation problem, J. Approx. Theory 13 (1975), 392-394.

[3] J. Diestel, H. Jarchow and A. Tonge, Absolutely Summing Operators, Cambridge Univ. Press, 1995.

[4] P. Enflo, A counterexample to the approximation property in Banach spaces, Acta Math. 130 (1973), 309-317.

[5] A. Grothendieck, Produits tensoriels topologiques et espaces nucléaires, Mem. Amer. Math. Soc. 16 (1955).

[6] J. Lindenstrauss and L. Tzafriri, Classical Banach Spaces I. Sequence Spaces, Springer, Berlin, 1977.

[7] A. Pietsch, Absolute p-summierende Abbildungen in normierten Räumen, Studia Math. 28 (1967), 333-353.

[8] - Operator Ideals, North-Holland, Amsterdam, 1980.

[9] O. I. Reĭnov, Disappearance of tensor elements in the scale of p-nuclear operators, in: Operator Theory and Function Theory 1, Leningrad. Univ., Leningrad, 1983, 145-165 (in Russian).

[10] —, Simple proof of two theorems of A. Grothendieck, Vestnik Leningrad Univ. Mat. Mekh. Astronom. 1983, no. 2, 115-116 (in Russian).

[11] P. D. Saphar, Produits tensoriels d'espaces de Banach et classes d'applications linéaires, Studia Math. 38 (1970), 71-100.

[12] - Hypothèse d'approximation à l'ordre $p$ dans les espaces de Banach et approximation d'applications p absolument sommantes, Israel J. Math. 13 (1972), 379-399.

Deba P. Sinha

Department of Mathematics

Dyal Singh College

University of Delhi

Lodi Road, New Delhi 110003

India

E-mail: dpsinha@nda.vsnl.net.in
Anil K. Karn

Department of Mathematics

Deen Dayal Upadhyaya College University of Delhi Karam Pura, New Delhi 110015 India E-mail: anilkarn@vsnl.net

Received June 27, 2000

Revised version January 29, 2001 